\title{
Emergency Peripartum Hysterectomy: A 10-Year Review
}

\author{
Joana Ferreira Carvalho, Adelaide Cubal, Sílvia Torres, \\ Fernanda Costa, and Olímpia do Carmo
}

Obstetrics and Gynaecology Department, Tâmega and Sousa Hospital Center, 4564-007 Penafiel, Portugal

Correspondence should be addressed to Joana Ferreira Carvalho, joanaifcarvalho@gmail.com

Received 28 August 2012; Accepted 11 September 2012

Academic Editors: A. K. Attri, D. Doll, O. Karcioglu, and F. Lateef

Copyright (C 2012 Joana Ferreira Carvalho et al. This is an open access article distributed under the Creative Commons Attribution License, which permits unrestricted use, distribution, and reproduction in any medium, provided the original work is properly cited.

\begin{abstract}
Background. Emergency peripartum hysterectomy (EPH), although rare in modern obstetrics, remains a life-saving procedure in cases of severe hemorrhage. Purpose. To determine the incidence, risk factors, indications, outcomes, and complications of EPH performed in a tertiary teaching hospital and to compare the results with other reports in the literature. Methods. The medical records of 13 patients who had undergone EPH, between January 2000 and December 2010, were reviewed retrospectively. Maternal characteristics and characteristics of the present pregnancy and delivery, hysterectomy indications, operative complications, postoperative conditions, and maternal and neonatal outcomes were evaluated. Results. There were 13 EPHs out of 31.767 deliveries, a rate of 0.41 per 1,000 deliveries. Eight hysterectomies were performed after cesarean delivery and five after vaginal delivery. The most common indication for hysterectomy was uterine atony (10/13), followed by placenta previa $(2 / 13)$. There were one case of intraoperative bladder injury and one case of relaparotomy because of hemoperitoneum. We had one maternal death because of septic shock. There were no cases of neonatal morbidity and mortality. Conclusion. Postpartum hemorrhage is one of the leading causes of maternal mortality and morbidity and represents the most challenging complication that an obstetrician will face.
\end{abstract}

\section{Introduction}

Emergency peripartum hysterectomy (EPH) is an uncommon obstetric procedure, usually performed as a life-saving measure in cases of intractable obstetric hemorrhage. [1-3] It was first proposed in 1869 but with no desirable results [4]. However, seven years later (1876), the first cesarean subtotal hysterectomy was carried out successfully, with the result that both the mother and the baby survived [5]. In modern obstetrics, the overall incidence of EPH is $0.05 \%$, but there are considerable differences in incidence in different parts of the world, depending on modern obstetric services, standards and awareness of antenatal care, and the effectiveness of family planning activities of a given community [6]. The incidence of peripartum hysterectomy in the literature is reported as $0.24,0.77,2.3$, and 5.09 per 1,000 deliveries by Sakse et al. [7], Whiteman et al. [8], Bai et al. [9] and Zeteroglu et al. [10], respectively. However, there is a lack of Portuguese data on EPH. To our knowledge, there is no Portuguese information on EPH.
Severe postpartum hemorrhage was reported to occur in $6.7 / 1,000$ deliveries worldwide. It is one of the leading causes of maternal mortality and morbidity and represents the most challenging complication that an obstetrician will face [11]. The main causes of the uncontrollable hemorrhage necessitating an EPH have changed since the 1980s [2]. Uterine atony and rupture have been overtaken by abnormal placentation in many studies. This is not only because of improved conservative management of uterine atony and a reduced incidence of uterine rupture due to the extensive use of the lower uterine segment incision in preference to the upper uterine segment incision for cesarean section (CS), but also because of an actual increase in the incidence of the morbidly adherent placenta. Abnormal placentation, which refers to both placenta previa and the morbidly adherent placenta, is thought to be increasing because of the rising rate of CS. Studies have consistently demonstrated that previous CS increases the risk of EPH and abnormal placentation is associated with a previous uterine scar. It is also established 
that the risk of EPH increases with the number of previous CS. Other factors that have been associated with EPH include advanced maternal age, multiparity, multiple gestations, and gestational diabetes. [2, 12-16] Conservative treatment of postpartum hemorrhage includes uterotonics (oxytocin, ergotamine), uterine massage, uterine artery embolization, uterine packing, pelvic vessel ligation, B-Lynch suture, multiple square sutures, and recombinant-activated factor VII [17]. The most severe complication of hemorrhage is maternal death, whose risk is estimated to be approximately 1 in 100,000 deliveries in developed countries and has been increasing. This risk is as high as 1 in 1,000 deliveries in developing countries. Other maternal complications of postpartum hemorrhage include hypovolemic shock, disseminated intravascular coagulopathy, renal failure, hepatic failure, and adult respiratory distress syndrome (ARDS) [18, 19].

The objectives of this retrospective study are to examine the incidence, risk factors, indications, outcomes and complications of EPH performed in a tertiary teaching hospital, between January 2000 and December 2010, and to compare the results with other reports in the literature. This would help highlight the lack of availability and utilization of antenatal services, identify avoidable factors, and stress the need to organize health care services so as to improve maternal and fetal outcome.

\section{Materials and Methods}

This study was a case series study. Medical and pathological records of the patients who had undergone emergency hysterectomy following vaginal or cesarean delivery due to postpartum hemorrhage, between January 2000 and December 2010, in a tertiary teaching hospital, were reviewed retrospectively. Cases were ascertained via a review of the hospital obstetric database and we ensure that no cases were missed by also checking operating theatre and pathology records. Emergency peripartum hysterectomy was defined as a hysterectomy performed in a life-threatening condition of postpartum hemorrhage. All deliveries were performed after 24 weeks of gestation, and the hysterectomy was performed shortly (within hours) after delivery. Both medical and surgical modalities were used to control the hemorrhage before hysterectomy. Information obtained from the medical records included demographic details, previous obstetric history, details of the current pregnancy and delivery, postpartum hemorrhage, indications for peripartum hysterectomy, outcomes of hysterectomy as intraoperative and postoperative complications, length of hospital stay, amount of blood transfused, and neonatal outcomes. Maternal complications such as maternal death and serious hemorrhagic, neurological, urological, infectious, respiratory, renal, and thromboembolic complications were also checked.

All statistical analysis was done using the SPSS for Windows release 11.5 packet program. Student's $t$-test and chi-squared test were used. Alpha was set at 0.05 for the statistical significance level. Frequencies and percentages were given as descriptive statistics.

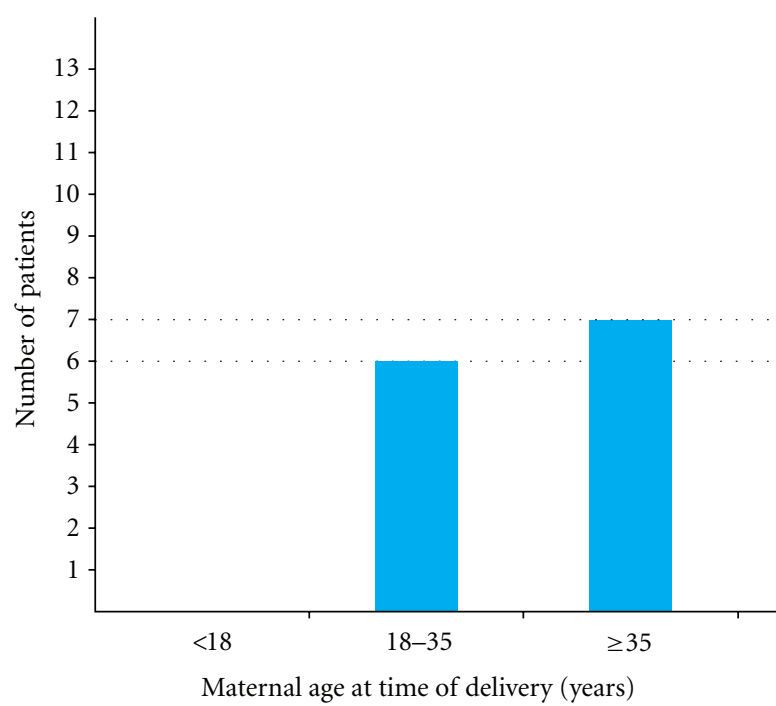

Figure 1: Maternal age at time of delivery.

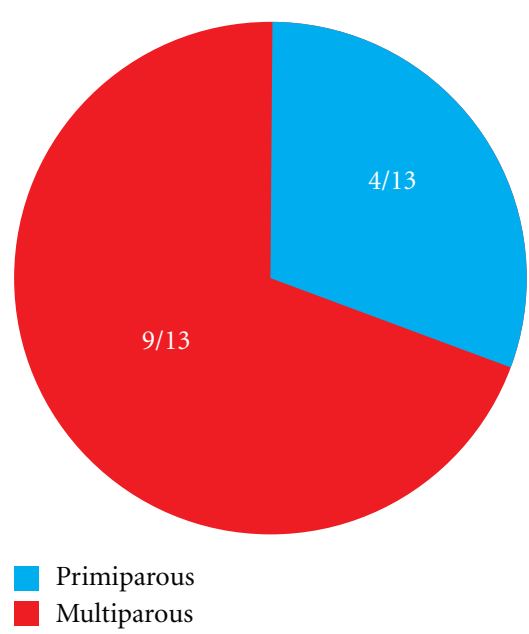

Figure 2: Parity of the patients who had undergone EPH.

\section{Results}

During the 10-year study period, a total of 31.767 women delivered. There were 13 emergency peripartum hysterectomies; 8 of the EPH were performed after cesarean delivery and 5 after vaginal delivery.

Maternal characteristics and obstetric data are shown in Figures 1 to 4 .

The mean maternal age was 32 years. Majority of patients (7/13) were $\geq 35$ years of age, and the others (6/13) were in the age group of $18-35$ years. There were no cases with maternal age $<18$ years (Figure 1 ).

The majority of women were multiparous (9/13); of these, only one case was a grandmultiparous woman (parity $>3$ ). There were 4 primiparous women (Figure 2).

Mean gestational age was 39 weeks (range 37-41) (Figure 3).

In Figure 4 we present obstetric data in relation to the type of delivery and its indications. After analysing this chart, we can conclude that the majority of cases $(8 / 13)$ 


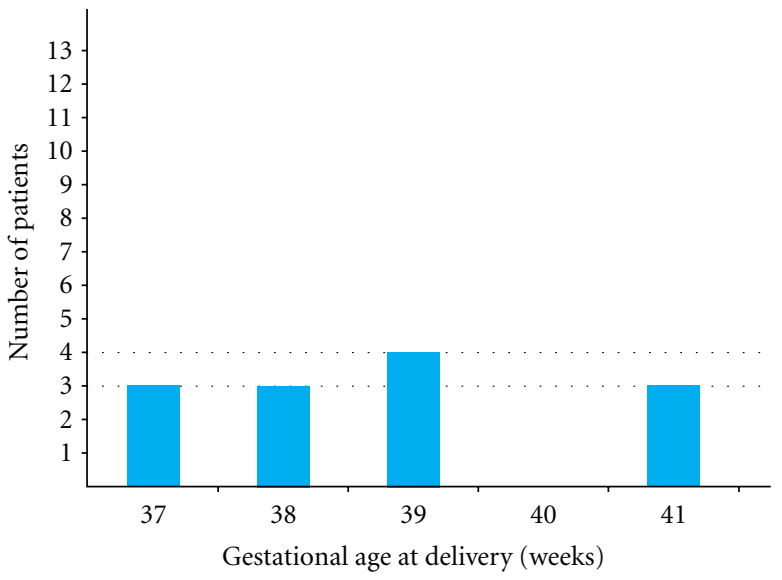

Figure 3: Gestational age at delivery.

were cesarean delivery, followed by vaginal delivery in the others (5/13). Of these, there were 3 cases of vaginal delivery with vacuum device, whose indications were absence of descent of the presentation in 2 cases and nonreassuring fetal status in 1 case. In relation to cesarean deliveries, 5/8 were elective cesareans and 3/8 were intrapartum cesareans. The most frequent indications for elective cesareans were placenta previa (2 cases), abnormal presentation (1 case), history of cesarean section (1 case), and ophthalmic maternal pathology with formal indication for cesarean delivery (1 case). The intrapartum cesareans were made because of dystocia ( 2 cases) and nonreassuring fetal status ( 1 case).

Thirteen hysterectomies were performed due to intractable obstetric hemorrhage that was unresponsive to conservative management, representing an incidence of 0.41 per 1,000 deliveries $(0.04 \%)$. To avoid hysterectomy, pharmacological agents and surgical procedures were used to control hemorrhage. All patients received oxytocin and ergotamine derivative. B-Lynch suture was performed in 3 cases, uterine packing was made in 3 other cases, pelvic vessel ligation was made in 1 case and multiple square sutures in another one. Indications for emergency peripartum hysterectomy are shown in Figure 5. The most common indication for EPH was uterine atony (10/13), followed by placenta previa and/or accreta (2/13); there was one case of uterine rupture.

We analysed the risk factors that predispose to EPH in our population and they are shown in Figure 6.

In relation to cases of uterine atony (10/13), we can conclude that 6 of these women were multiparous women and in 7 cases the labor was induced with prostaglandins and/or oxytocin. In 6 of these 10 cases, the delivery was performed by cesarean. Another prevalent risk factor identified in the group of cases of uterine atony was maternal age $\geq 35$ years ( 4 cases). From the analysis of this chart, we can rapidly conclude that the 2 cases of placenta previa and/or accreta occurred in women with $\geq 35$ years of age and with a history of cesarean section. The case of uterine rupture occurred in a multiparous women aged $\geq 35$ years with a previous cesarean delivery and in whose actual labor oxytocin was used. The delivery was with vacuum device and the newborn was a macrosomic one.
TABLE 1: Operative complications, postoperative conditions, and maternal outcomes. Neonatal outcomes.

\begin{tabular}{lc}
\hline Maternal and neonatal complications & No. of cases \\
\hline Maternal morbidity & \\
$\quad$ Intraoperative bladder injury & $1 / 13$ \\
Relaparotomy for hemoperitoneum & $1 / 13$ \\
Blood transfusions & $13 / 13$ \\
$\quad$ Disseminated intravascular coagulopathy & $4 / 13$ \\
Maternal mortality & \\
$\quad$ Maternal death because of postoperative septic & $1 / 13$ \\
$\quad$ shock & \\
Neonatal complications & \\
$\quad$ Neonatal morbidity and mortality & $0 / 13$ \\
\hline
\end{tabular}

The operative complications, postoperative conditions and maternal outcomes are shown in Table 1.

There was one case of intraoperative bladder injury. This patient had a previous cesarean section. There was one case of relaparotomy because of persistent intra-abdominal bleeding, with good resolution. All patients received blood transfusions, with the median number of units of blood transfused being 9 (range 4-35). We had 4 cases of disseminated intravascular coagulopathy that reversed with prompt management. The median postoperative hospital stay was 6 (range 5-25) days. There was one maternal death because of infectious postoperative complications with the development of a septic shock.

Neonatal outcomes are also shown in Table 1. The mean birth weight of the 13 infants was $3491 \mathrm{~g}$ (range 2550-4390). The proportion of female babies $(8 / 13)$ was higher than that of male babies $(5 / 13)$. There were no cases of neonatal morbidity and mortality.

\section{Discussion}

Despite advances in medicine and surgery, postpartum hemorrhage remains one of the leading causes of maternal morbidity and mortality. Peripartum hysterectomy is performed in the treatment of a life-threatening obstetric hemorrhage that cannot be controlled by conventional methods. The reported incidence of emergency peripartum hysterectomy varies from 0.24 to 5.09 per 1,000 deliveries in the literature. Our incidence of 0.41 per 1,000 deliveries $(0.04 \%)$ is in agreement with the recent studies. Zeteroglu et al. reported the incidence of EPH in a teaching hospital as 5.09/1,000 deliveries, which is higher than that of other studies [10].

In our study, majority of patients who underwent EPH were in age group $\geq 35$ years and were multipara. Similar trend was observed by Amad and Mir [20] and Barclay et al. [21]. Other risk factors for EPH, like previous cesarean birth, induced labor, current cesarean delivery, and abnormal placental implantation and invasion, were similar to the literature [22].

The most frequent indication for EPH in the present study was uterine atony, followed by morbid adherence of 


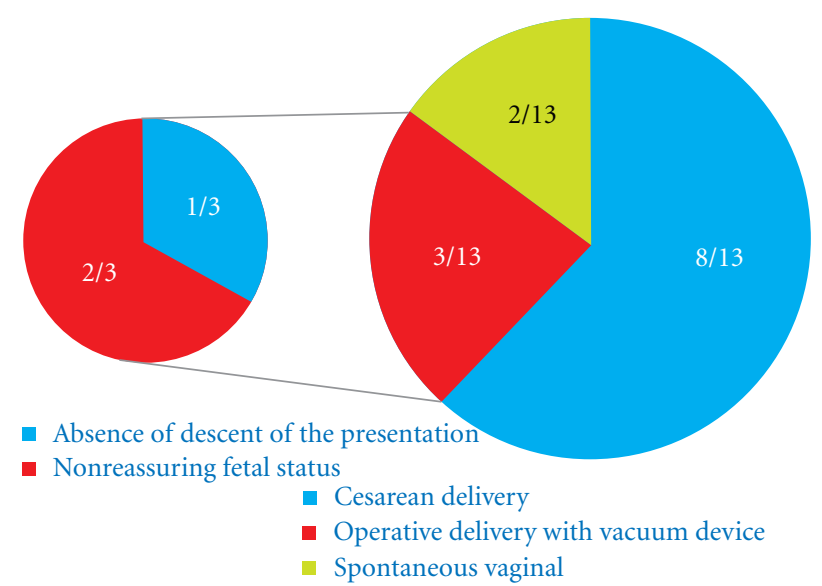

(a)

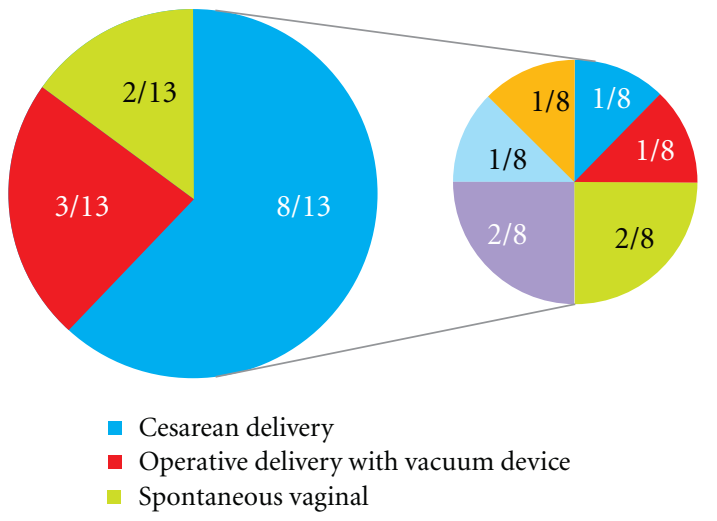

$\left.\begin{array}{l}\left.\begin{array}{l}\text { History of cesarean section } \\ \text { and 41-weeks gestational age } \\ \text { Ophthalmic maternal pathology } \\ \text { with formal indication for } \\ \text { cesarean delivery } \\ \text { - Placenta previa } \\ \text { Abnormal presentation }\end{array}\right] \begin{array}{l}\text { Elective } \\ \text { cesarean }\end{array} \\ \text { - Dystocia } \\ \text { - Nonreassuring fetal status }\end{array}\right\} \begin{aligned} & \text { Intra-partum } \\ & \text { cesarean }\end{aligned}$

(b)

FIgUre 4: Type of delivery and its indications.

placenta and uterine rupture. There has been a significant change in the indication of EPH over time and from one region to another. Traditionally, uterine atony was the most common indication for hysterectomy. Recent studies have indicated that abnormal placentation is replacing uterine atony as the most common indication for EPH [4]. In 1984, Stanco et al. reported that $43.4 \%$ of their emergency hysterectomies were done because of uterine atony, while $33.9 \%$ were due to placenta previa with accreta. A study from the same institution in 1993 stated that their primary indication was placenta accreta, the problem in $45 \%$ of cases, followed by uterine atony, with $20 \%$ [23]. Baskett reported that the main indications for hysterectomy were abnormal placentation (50\%) and atonic postpartum hemorrhage (32.8\%) [13]. This study demonstrates that our most frequent indication for EPH still remains uterine atony, followed by placenta previa, a feature that can be explained by our low rate of cesarean delivery. In fact, we are the Portuguese obstetric department with the lowest rate of cesarean delivery in the last five years (rate of 26.0\%). Also, despite the above reports, similar results to our study have been described by Özden et al. and that was also explained by the low rate of cesarean delivery [24]. From all this data, we can conclude that there is a considerable variability in the indications of $\mathrm{EPH}$ worldwide, and it varies with obstetric practice in each center.
Peripartum hysterectomy is associated with high complication rates, mainly due to the need for massive blood transfusions, coagulopathy, and injury of the urinary tract, and it is also associated with the need for reexploration because of persistent bleeding and febrile morbidity [2, 25, 26]. All of our patients received blood transfusions and $>98 \%$ of them had over two units of blood. Bladder injury was found in 1 patient, and this patient had a previous cesarean delivery. Thus, urological injuries appear to be related to scarring and secondary adhesion of the vesicouterine space following previous cesarean section. In comparison with Smith's 6\%, Kwee's 15\%, Yucel's 8.8\%, Zeteroglu's 12.5\%, and Zelop's $9 \%$, our urinary tract injury rate is $7.7 \%$. [2, 10, $17,26,27]$ Reexploration was performed in 1 case $(7.7 \%)$ for persistent postoperative bleeding, compared with Smith's $11 \%$, Kwee's 25\%, Zeteroglu's 12.5\%, and Ozden's 6.8\% $[2,10,17,24]$. In our series, 4 women $(30.7 \%)$ developed disseminated intravascular coagulopathy, lower than the $33 \%$ rate previously reported by Smith and Mousa and Lau et al. $[17,22]$. The febrile morbidity rate of $7.7 \%$ is lower than that of their studies $[14,17,28]$. There was one maternal death $(7.7 \%)$ in our study. Lower rates of 4 and $4.5 \%$ were cited by Kwee et al. and Zorlu et al. and much higher rates of 20 and $23.8 \%$ were found by Hamsho and Alsakka and Umezurike et al. [2, 29-31]. Our low mortality rate may be related to a high rate of antenatal followup and an 


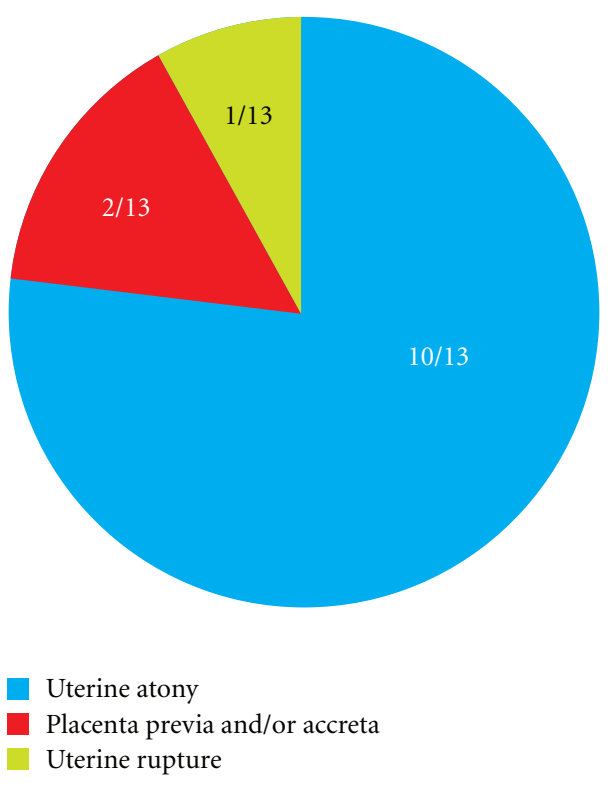

Figure 5

FIGURE 5: Indications for emergency peripartum hysterectomy.

optimal obstetric intervention in the cases of EPH in our department. Our results confirm the previous observations that $\mathrm{EPH}$ is associated with high operative and postoperative complications rates.

There are numerous risk factors that can contribute for this entity and recognizing and assessing patients at risk is very important. Also, appropriate management of cases of postpartum hemorrhage is an important issue. Ideally each labor and delivery unit has a postpartum hemorrhage protocol for patients with estimated blood loss exceeding a predefined threshold (often $1000 \mathrm{~mL}$ ). These protocols provide a standardized approach to evaluating and monitoring the patient, notifying a multidisciplinary team, and treatment. In our department, we have implemented the following sequential steps in managing postpartum hemorrhage.

(i) Assemble team and notify appropriate departments (obstetrics, nursing, anesthesiology, blood bank, and laboratory).

(ii) Initiate uterine massage and establish large bore (two 14-gauge) intravenous access.

(iii) Administer oxygen (8 liters/minute) by face mask. Anesthesia team should evaluate airway and breathing, intubate if indicated.

(iv) Fluid resuscitation: infuse crystalloid (at least 3 liters for each liter of estimated blood loss).

(v) Transfusion: if hemodynamics do not improve with 2 to 3 liters of crystalloid administration and bleeding continues, administer blood products, initially 2 units packed red blood cells. For massive transfusion, administer red blood cells, fresh frozen plasma and apheresis platelets in a ratio of $6: 4: 1$ or $4: 4: 1$. (vi) Identify and treat specific causes of bleeding: inspect the vagina and cervix for lacerations and repair them as necessary; evacuate any retained products of conception; replace uterus if inverted.

(vii) Administer uterotonic drugs to reverse atony: it should be possible to determine within 30 minutes whether uterotonic treatment will reverse atony. If does not, prompt invasive intervention is usually warranted.

(a) Begin with oxytocin 10 units intravenously, followed by 40 units in $500 \mathrm{~mL}$ of normal saline. Using an intravenous infusion pump, start at $125 \mathrm{~mL} / \mathrm{h}$. Adjust rate to achieve and maintain uterine contraction. Expect rapid response.

(b) Avoid rapid intravenous bolus injection of oxytocin.

(c) If no intravenous access, give 10 units intramuscularly; expect response within 3 to 5 minutes.

(d) There are no absolute contraindications to oxytocin for postpartum hemorrhage.

(viii) Add prostaglandin.

(a) $1000 \mathrm{mcg}$ sulprostone in $500 \mathrm{~mL}$ of normal saline intravenously at a rate of $125 \mathrm{~mL} / \mathrm{h}$. If necessary, adjust rate to a maximum of $500 \mathrm{~mL} /$ $\mathrm{h}$ and return to initial dose after stabilization. Avoid in women with asthma/bronchospasm or hypertension.

(b) Misoprostol ( $\left.\mathrm{PGE}_{1}\right) 1000 \mathrm{mcg}$ rectally as a single dose. Can be given to women with asthma/ bronchospasm and hypertension. Monitor for pyrexia.

(ix) Tamponade bleeding from the uterine cavity. Options include Sengstaken-Blakemore tube, and Foley catheter balloon.

(x) Perform laparotomy if the above measures fail. Surgical approaches that are quick, relatively easy and effective should be tried first. In utilizing these measures, the surgeon should be cognizant of the amount of blood and the stability of the patient and should perform hysterectomy rather than resort to temporizing measures if her cardiovascular status is unstable or if it appears that the anesthesiologist will not be able to keep up with her fluid needs. Options include:

(a) ligate bleeding sites;

(b) perform uterine artery ligation;

(c) perform hypogastric artery ligation;

(d) place a B-Lynch stitch or other uterine compression suture;

(e) perform hysterectomy-Hysterectomy is the last resort, but should not be delayed in women who have disseminated intravascular coagulation and require prompt control of uterine hemorrhage to prevent death; 


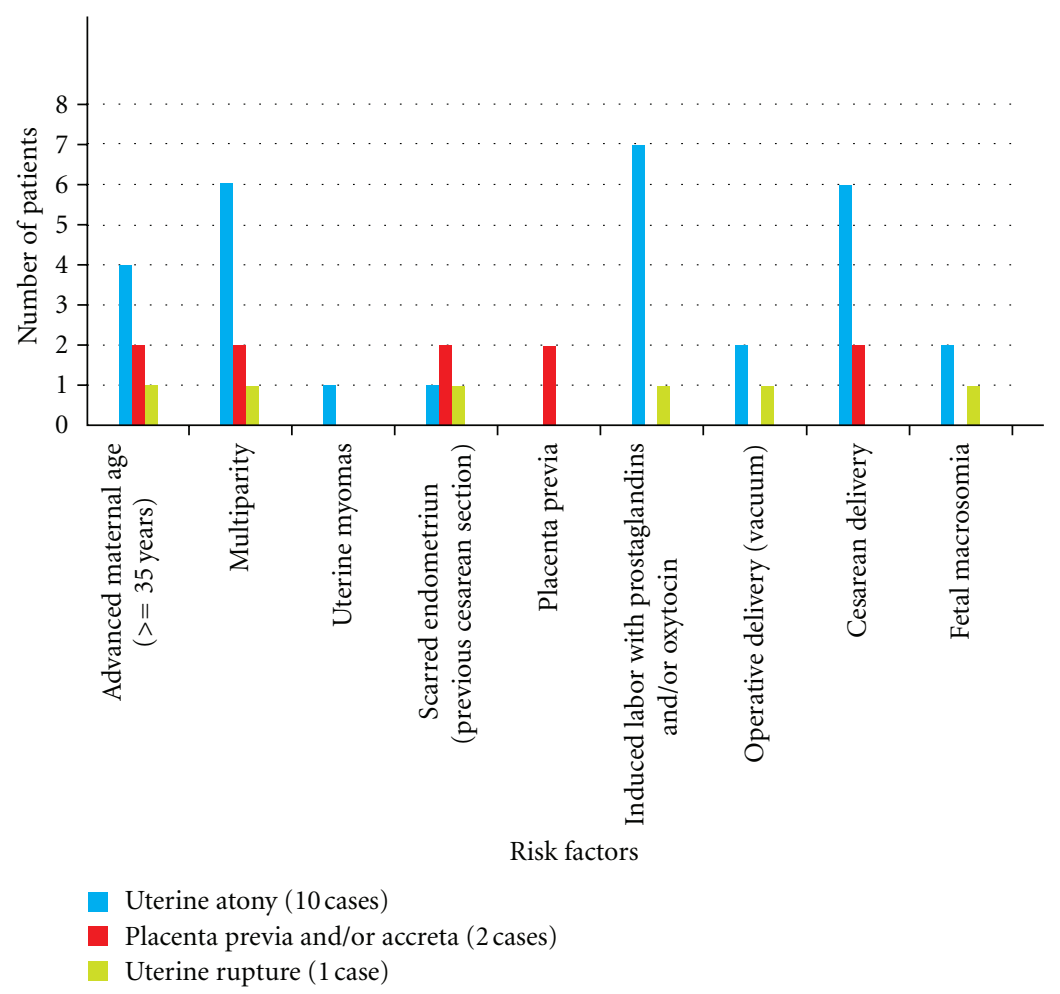

FIGURE 6: Risk factors.

(f) suture deep pelvic bleeders;

(g) tamponade pelvic bleeding with pelvic packing.

In conclusion, the risk factors associated with emergency peripartum hysterectomy should be identified antenatally and the high risk group of women should be delivered by skilled birth attendants and following protocols of action, measures that can contribute to reduce the high maternal morbidity and mortality associated to EPH. Also, cesarean delivery should be performed only when exclusively necessary, in appropriate clinical settings and by experienced surgeons when such risk factors are identified.

\section{Conflict of Interests}

The authors declare no conflict of interests.

\section{References}

[1] A. C. Rossi, R. H. Lee, and R. H. Chmait, "Emergency postpartum hysterectomy for uncontrolled postpartum bleeding: a systematic review," Obstetrics and Gynecology, vol. 115, no. 3, pp. 637-644, 2010.

[2] A. Kwee, M. L. Bots, G. H. A. Visser, and H. W. Bruinse, "Emergency peripartum hysterectomy: a prospective study in the Netherlands," European Journal of Obstetrics Gynecology and Reproductive Biology, vol. 124, no. 2, pp. 187-192, 2006.

[3] M. E. Akar, E. S. Yilmaz, B. Yuksel, and Z. Yilmaz, "Emergency peripartum hysterectomy," European Journal of Obstetrics Gynecology and Reproductive Biology, vol. 113, no. 2, pp. 178$181,2004$.
[4] G. Daskalakis, E. Anastasakis, N. Papantoniou, S. Mesogitis, M. Theodora, and A. Antsaklis, "Emergency obstetric hysterectomy," Acta Obstetricia et Gynecologica Scandinavica, vol. 86, no. 2, pp. 223-227, 2007.

[5] D. W. Sturdee and D. I. Rushton, "Caesarean and post-partum hysterectomy 1968-1983," British Journal of Obstetrics and Gynaecology, vol. 93, no. 3, pp. 270-274, 1986.

[6] E. H. Park and B. P. Sachs, “. Postpartum haemorrhage and other problems of third stage," in High Risk PregnancyManagement Options, D. K. James, P. J. Steer, C. P. Weiner, and B. Gonik, Eds., pp. 1231-1246, W.B. Saunders, Philadelphia, Pa, USA, 2nd edition, 1999.

[7] A. Sakse, T. Weber, C. Nickelsen, and N. J. Secher, "Peripartum hysterectomy in Denmark 1995-2004," Acta Obstetricia et Gynecologica Scandinavica, vol. 86, no. 12, pp. 1472-1475, 2007.

[8] M. K. Whiteman, E. Kuklina, S. D. Hillis et al., "Incidence and determinants of peripartum hysterectomy," Obstetrics and Gynecology, vol. 108, no. 6, pp. 1486-1492, 2006.

[9] S. W. Bai, H. J. Lee, J. S. Cho, Y. W. Park, S. K. Kim, and K. H. Park, "Peripartum hysterectomy and associated factors," Journal of Reproductive Medicine for the Obstetrician and Gynecologist, vol. 48, no. 3, pp. 148-152, 2003.

[10] S. Zeteroglu, Y. Ustun, Y. Engin-Ustun, G. Sahin, and M. Kamaci, "Peripartum hysterectomy in a teaching hospital in the eastern region of Turkey," European Journal of Obstetrics Gynecology and Reproductive Biology, vol. 120, no. 1, pp. 5762, 2005.

[11] M. Waterstone, S. Bewley, and C. Wolfe, "Incidence and predictors of severe obstetric morbidity: case-control study," British Medical Journal, vol. 322, no. 7294, pp. 1089-1093, 2001. 
[12] S. Bakshi and B. A. Meyer, "Indications for and outcomes of emergency peripartum hysterectomy: a five-year review," Journal of Reproductive Medicine for the Obstetrician and Gynecologist, vol. 45, no. 9, pp. 733-737, 2000.

[13] T. F. Baskett, "Emergency obstetric hysterectomy," Journal of Obstetrics and Gynaecology, vol. 23, no. 4, pp. 353-355, 2003.

[14] D. O. Selo-Ojeme, P. Bhattacharjee, N. F. Izuwa-Njoku, and R. A. Kadir, "Emergency peripartum hysterectomy in a tertiary London hospital," Archives of Gynecology and Obstetrics, vol. 271, no. 2, pp. 154-159, 2005.

[15] F. Kayabasoglu, K. Guzin, S. Aydogdu, S. Sezginsoy, L. Turkgeldi, and G. Gunduz, "Emergency peripartum hysterectomy in a tertiary Istanbul hospital," Archives of Gynecology and Obstetrics, vol. 278, no. 3, pp. 251-256, 2008.

[16] S. Wu, M. Kocherginsky, and J. U. Hibbard, "Abnormal placentation: twenty-year analysis," American Journal of Obstetrics and Gynecology, vol. 192, no. 5, pp. 1458-1461, 2005.

[17] J. Smith and H. A. Mousa, "Peripartum hysterectomy for primary postpartum haemorrhage: incidence and maternal morbidity," Journal of Obstetrics and Gynaecology, vol. 27, no. 1, pp. 44-47, 2007.

[18] H. A. Mousa and S. Walkinshaw, "Major postpartum haemorrhage," Current Opinion in Obstetrics and Gynecology, vol. 13, no. 6, pp. 595-603, 2001.

[19] M. Hall, "Haemorrhage," in Why Mothers Die 2000-2002, Royal College Of Obstetricians and Gynecologists, London, UK, 2004

[20] S. N. Amad and I. H. Mir, "Emergency peripartum hysterectomy: experience at Apex Hospital of Kashmir Valley," Internet Journal of Gynecology \& Obstetric, vol. 8, no. 2, 2007, http://www.ispub.com/journal/the_internet_journal_of_gynec ology_and_obstetrics/volume_8_number_2_5/article/emergenc y_peripartum_hysterectomy_experience_at_apex_hospital_of_k ashmir_valley.html.

[21] D. L. Barclay, B. L. Hawks, and D. M. Frueh, "Elective cesarean hysterectomy: a 5 year comparison with cesarean section," American Journal of Obstetrics and Gynecology, vol. 124, no. 8, pp. 900-911, 1976.

[22] W. C. Lau, H. Y. Fung, and M. S. Rogers, "Ten years experience of cesarean and postpartum hysterectomy in a teaching hospital in Hong Kong," European Journal of Obstetrics \& Gynecology and Reproductive Biology, vol. 74, pp. 133-137, 1997.

[23] L. M. Stanco, D. B. Schrimmer, R. H. Paul, and D. R. Mishell, "Emergency peripartum hysterectomy and associated risk factors," American Journal of Obstetrics and Gynecology, vol. 168, no. 3, pp. 879-883, 1993.

[24] S. Özden, G. Yildirim, T. Basaran, B. Gurbuz, and V. Dayicioglu, "Analysis of 59 cases of emergent peripartum hysterectomies during a 13-year period," Archives of Gynecology and Obstetrics, vol. 271, no. 4, pp. 363-367, 2005.

[25] D. H. Chestnut, R. D. Eden, S. A. Gall, and R. T. Parker, "Peripartum hysterectomy: a review of cesarean and postpartum hysterectomy," Obstetrics and Gynecology, vol. 65, no. 3, pp. 365-370, 1985.

[26] C. M. Zelop, B. L. Harlow, F. D. Frigoletto Jr., L. E. Safon, and D. H. Saltzman, "Emergency peripartum hysterectomy," American Journal of Obstetrics and Gynecology, vol. 168, no. 5, pp. 1443-1448, 1993.

[27] O. Yucel, I. Ozdemir, N. Yucel, and A. Somunkiran, "Emergency peripartum hysterectomy: a 9-year review," Archives of Gynecology and Obstetrics, vol. 274, no. 2, pp. 84-87, 2006.
[28] I. B. Engelsen, S. Albrechtsen, and O. E. Iversen, "Peripartum hysterectomy-incidence and maternal morbidity," Acta Obstetricia et Gynecologica Scandinavica, vol. 80, no. 5, pp. 409-412, 2001.

[29] C. G. Zorlu, C. Turan, A. Z. Işik, N. Danişman, T. Mungan, and O. Gökmen, "Emergency hysterectomy in modern obstetric practice. Changing clinical perspective in time," Acta Obstetricia et Gynecologica Scandinavica, vol. 77, no. 2, pp. 186-190, 1998.

[30] M. A. Hamsho and M. Alsakka, "Emergency obstetric hysterectomy in Qatar-a 20-year review," International Journal of Fertility and Women's Medicine, vol. 44, no. 4, pp. 209-211, 1999.

[31] C. C. Umezurike, P. A. Feyi-Waboso, and C. A. Adisa, "Peripartum hysterectomy in Aba southeastern Nigeria," Australian and New Zealand Journal of Obstetrics and Gynaecology, vol. 48 , no. 6, pp. 580-582, 2008. 


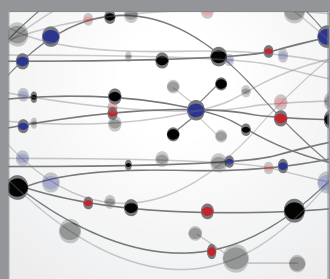

The Scientific World Journal
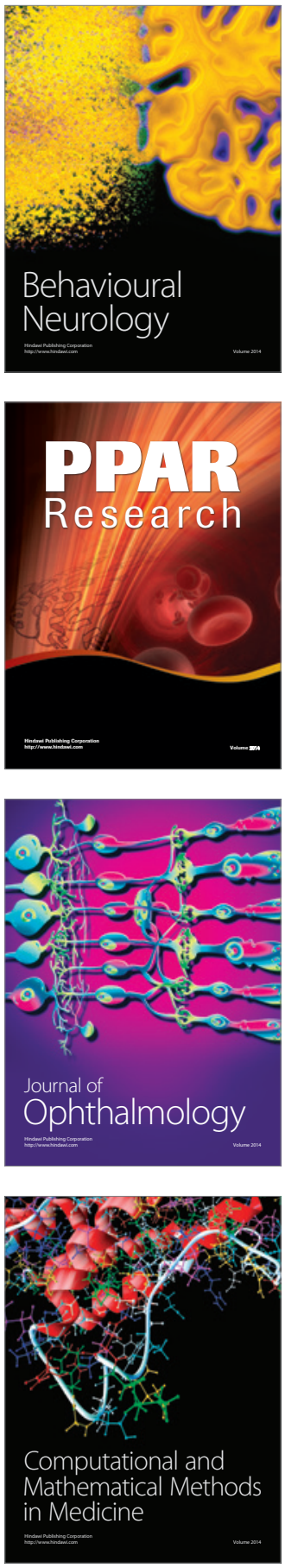

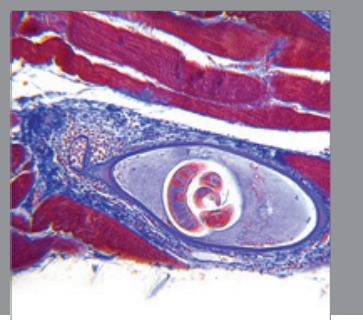

Gastroenterology

Research and Practice
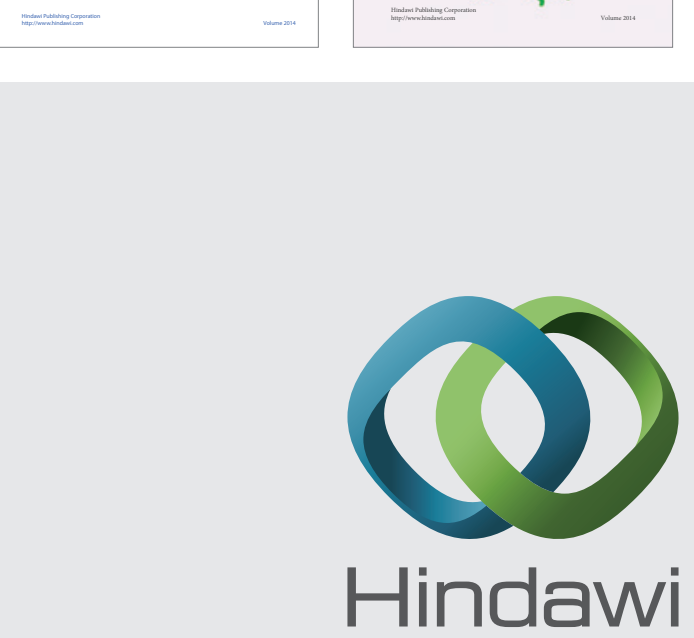

Submit your manuscripts at

http://www.hindawi.com
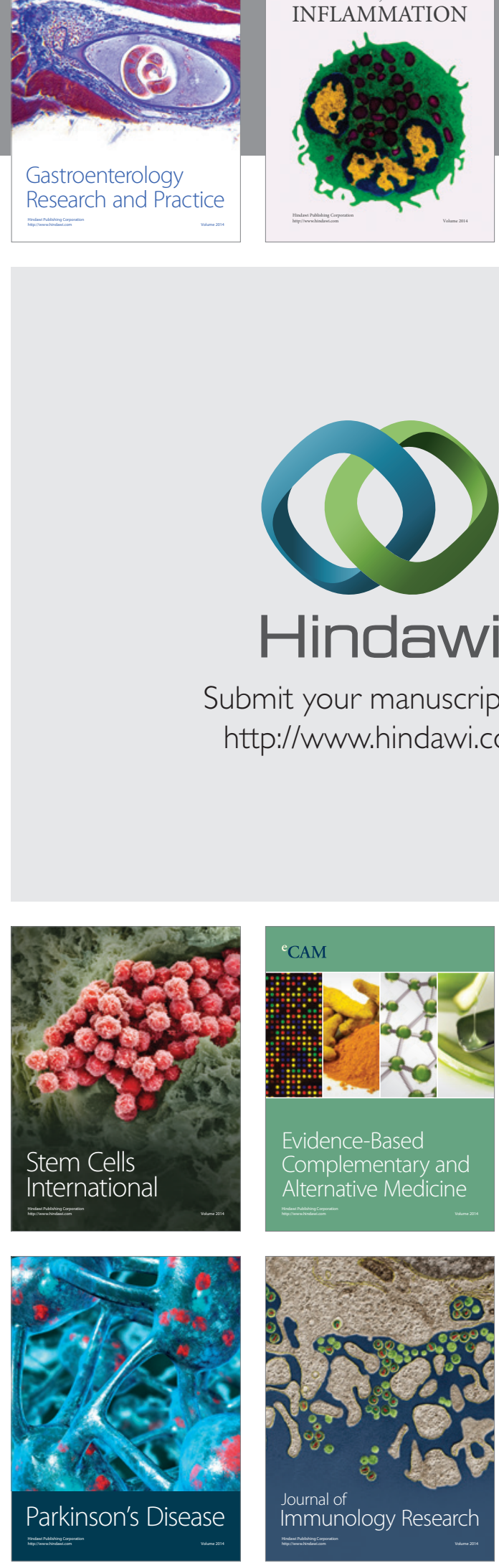

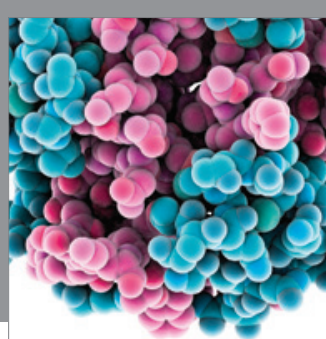

Diabetes Research
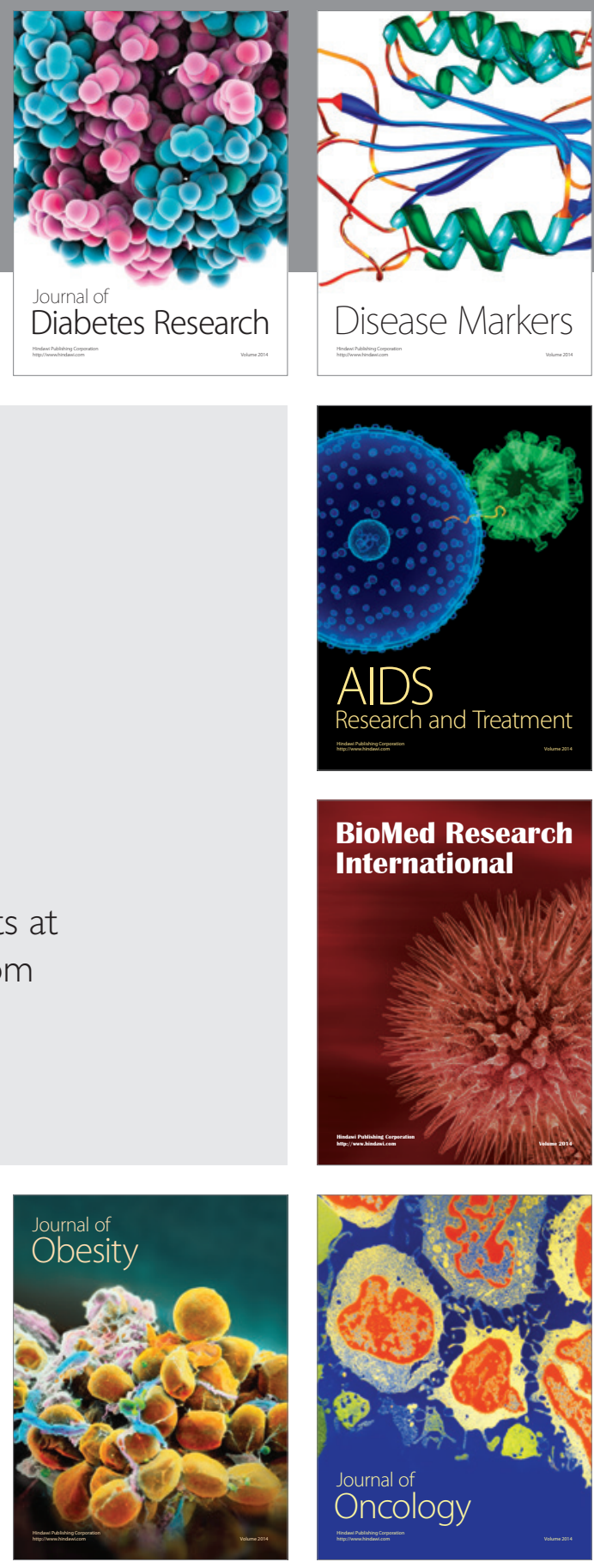

Disease Markers

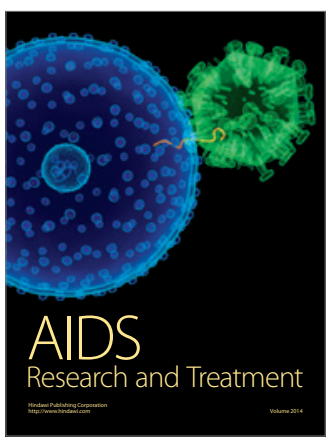

BioMed Research

International
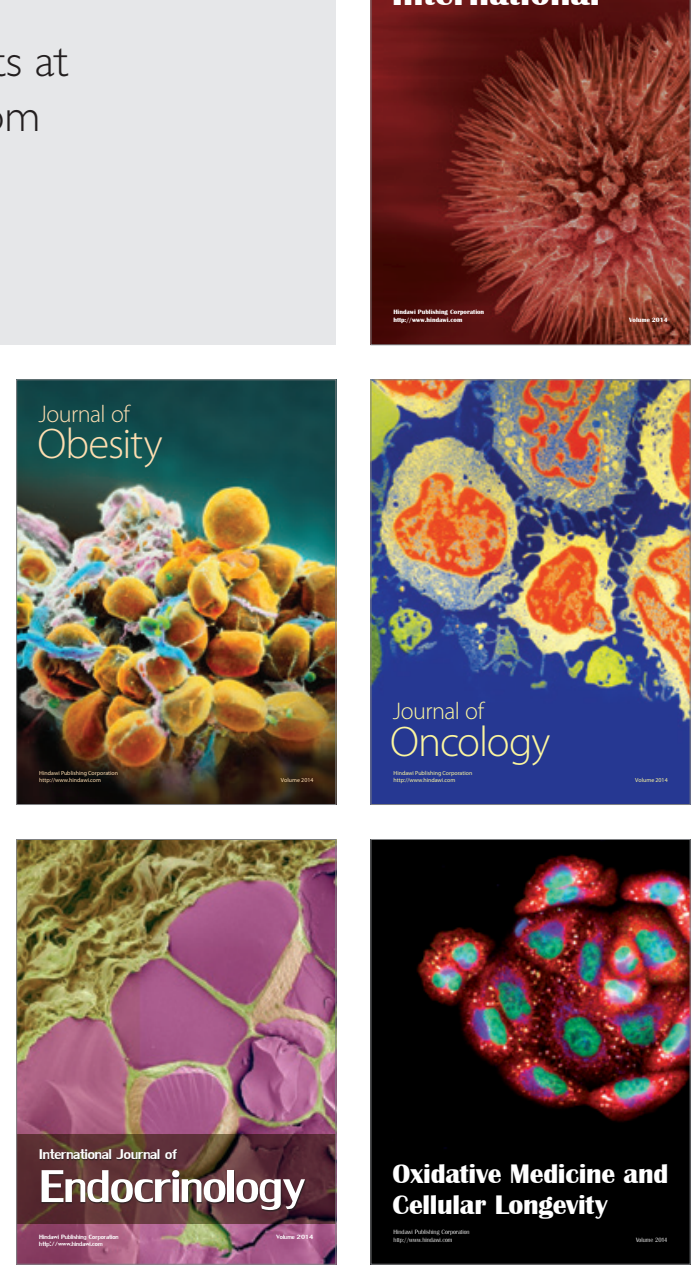